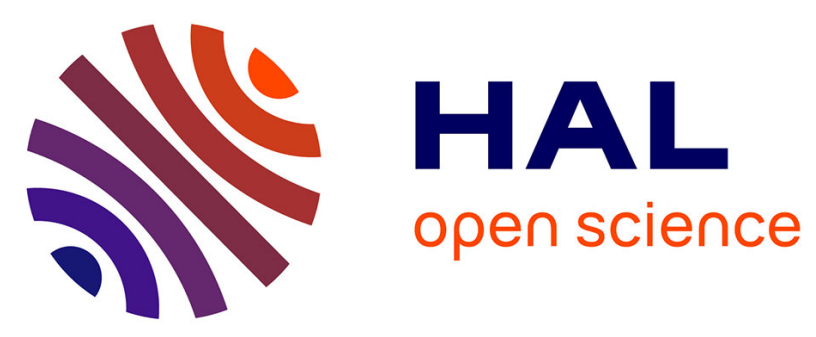

\title{
CURATING A LARGE-SCALE REGULATORY NETWORK BY EVALUATING ITS CONSISTENCY WITH EXPRESSION DATASETS
}

Carito Guziolowski, Jérémy Gruel, Ovidiu Radulescu, Anne Siegel

\section{- To cite this version:}

Carito Guziolowski, Jérémy Gruel, Ovidiu Radulescu, Anne Siegel. CURATING A LARGESCALE REGULATORY NETWORK BY EVALUATING ITS CONSISTENCY WITH EXPRESSION DATASETS. CIBB 2008: COMPUTATIONAL INTELLIGENCE METHODS FOR BIOINFORMATICS AND BIOSTATISTICS, Oct 2008, Salerno, Italy. pp.144-155. inria-00331385

\section{HAL Id: inria-00331385 \\ https://hal.inria.fr/inria-00331385}

Submitted on 16 Oct 2008

HAL is a multi-disciplinary open access archive for the deposit and dissemination of scientific research documents, whether they are published or not. The documents may come from teaching and research institutions in France or abroad, or from public or private research centers.
L'archive ouverte pluridisciplinaire HAL, est destinée au dépôt et à la diffusion de documents scientifiques de niveau recherche, publiés ou non, émanant des établissements d'enseignement et de recherche français ou étrangers, des laboratoires publics ou privés. 


\title{
CURATING A LARGE-SCALE REGULATORY NETWORK BY EVALUATING ITS CONSISTENCY WITH EXPRESSION DATASETS
}

\author{
Carito Guziolowski $^{(1) *}$, Jeremy Gruel ${ }^{(2)}$, Ovidiu Radulescu ${ }^{(3)}$, Anne Siegel $^{(4)}$
}

(1) Centre INRIA Rennes Bretagne Atlantique, IRISA

Campus de Beaulieu, 35042 Rennes, France

(2) INSERM U620

2 Av Pr. L. Bernard, 35043 Rennes, France

(3) Université de Rennes 1, IRMAR

Campus de Beaulieu, 35042 Rennes, France

(4) CNRS, UMR 6074, IRISA

Campus de Beaulieu, 35042 Rennes, France

Keywords: E. coli, transcriptional networks, expression prediction, stationary phase control.

\begin{abstract}
The analysis of large-scale regulatory models using data issued from genome-scale highthroughput experimental techniques is an actual challenge in the systems biology field. This kind of analysis faces three common problems: the size of the model, the uncertainty in the expression datasets, and the heterogeneity of the data. On that account, we propose a method that analyses large-scale networks with small - but reliable - expression datasets. Our method relates regulatory knowledge with heterogeneous expression datasets using a simple consistency rule. If a global consistency is found, we predict the changes in gene expression or protein activity of some components of the network. When the whole model is inconsistent, we highlight regions in the network where the regulatory knowledge is incomplete. Confronting our predictions with mRNA expression experiments allows us to determine the missing post-transcriptional interactions of our model. We tested this approach with the transcriptional network of E. coli. Sources and a working application of our method can be accessed on-line at: http://www.irisa.fr/symbiose/bioquali/
\end{abstract}

\section{Introduction}

Reconciling gene expression data with large-scale regulatory network structures is a subject of particular interest due to the urgent need of curating regulatory models that are likely to be incomplete and may contain errors. Based on the large amount of genome-scale data yielded by high-throughput experimental techniques, many data-driven approaches for reconstructing regulatory network structures have been proposed [21, 13,4]. Also, for some well studied organisms there are already large-scale models of regulations derived from literature curations or from computational predictions; as for example the RegulonDB database [26] for the bacteria E. coli. On account of this, large-scale models are presented as a compilation of interactions deduced from different methods or from experiments applied under different conditions. The accumulation of diverse sources in the construction of regulatory models may cause errors. A challenging solution is therefore to design automatic methods that integrate experimental datasets to known regulatory structures enabling biologists to conciliate heterogeneous data types, find inconsistencies, and refine and diagnose a regulatory model [18, 23, 22].

On the basis of these arguments, we propose an iterative method that integrates experimental data to a large-scale regulatory model and automatically evaluates the consistency of it in a reasonable time. We check the global consistency between the model and the expression dataset by applying a consistency rule to each product in the entire network. Precisely, the evaluation process consists

\footnotetext{
*Corresponding author contact details: e-mail carito.guziolowski@ irisa.fr, telephone +33(0)299847116
} 
on the following steps: finding inconsistencies between model and data, correcting the model, making model predictions, comparing model predictions with real measurements, and learning from the discrepancies found. We can concretely list the results obtained automatically as: ( $i$ ) detection of a region in the network that was inconsistent with the experimental data, and (ii) extension of the initial set of expression data (computational predictions). We applied our method to the transcriptional regulatory network of $E$. coli extracted from the RegulonDB database.

A first attempt to evaluate the consistency between a known regulatory model and experimental data at genomic-scale was proposed by [15]; the authors computed a local consistency measure for different types of network modules by analysing the expression datasets; afterwards, each regulatory interaction was assigned a level of consistency. This approach had the same initial direction as our approach, the type of method to evaluate the consistency was however different. Our approach relies on a global reasoning, and the same consistency rule is automatically applied to each module in the entire regulatory network. In [15], many expression profiles were taken into account to deduce a consistency relation between regulations and expression data. In our method, we only use one expression profile to establish this consistency; however, we retrieve from this analysis additional outcomes as the correction of the model either by detecting an inconsistency or by comparing the computational predictions with real data.

After the consistency check of a regulatory model with a small and reliable expression dataset we obtain two results. The first result is the curation of regulatory models by spotting regions where the model is inconsistent with the experimental data. Regarding the E. coli model, we concluded that its transcriptional interactions do not adequately explain the experimental observations. On that account, we detected specific post-transcriptional interactions in order to obtain a globally explained model by the experimental data. The second result, obtained after correcting the model, is the generation of gene-expression computational predictions. We may cite other methods that obtain gene-expression predictions after applying an artificial perturbation to in silico network models. For example [17] presented a similar rule approach aiming to test and refine a network of regulations. Their approach incorporated Protein-Protein interactions to their model at the cost of restraining the associated experiments to only genetic perturbations. The approach was mostly conceived to generate predictions of changes in gene-expression due to a particular genetic perturbation in a well studied but small pathway model that included physical interactions. A recent method that models and simulates microarray experiments using an steady state approach is the work of [25]; the same idea of computational predictions appears in this study but applied to a small genetic network and with the additional problem of requiring detailed information on kinetic parameters, regulatory structure, interaction constants, or transcription regulators levels. Our computational predictions, however, are the result of a consistency test performed previously to the entire network. As opposed to the mentioned methods, we do not simulate the response of our model to an artificial perturbation; we describe the (possibly huge) set of models that are consistent with the initial dataset, then we look for invariants in this set. Regarding the E. coli regulatory model, we calculated a set of gene-expression predictions and we validated them with real measurements obtaining an agreement of $80 \%$. This percentage can be comparable to the one obtained by other methods working on E. coli data [7, 6, 11], and considerable, since we used only a transcriptional model without including metabolic regulations.

\section{Approach}

\subsection{Global approach}

We analysed regulatory networks formed by the interactions among certain molecules. As molecules we refer to genes, sigma factors, active proteins, or protein complexes; and as interactions to ProteinDNA, Sigma-gene, and complex formation. Even so, any kind of interactions may be studied as long as we can map them as influence relations, i.e. A influences $B$ if increasing or decreasing $A$ 's concentration causes a change in B's concentration. Molecules that hold influence relations form an 
influence network, the central object of this study (Fig. 1).

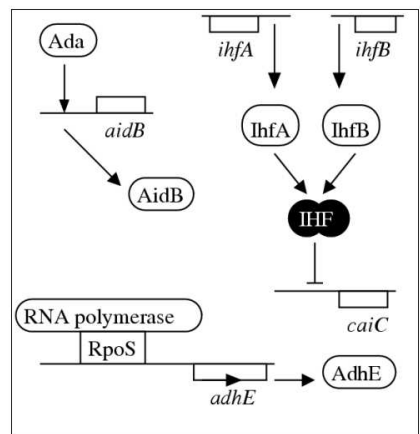

A

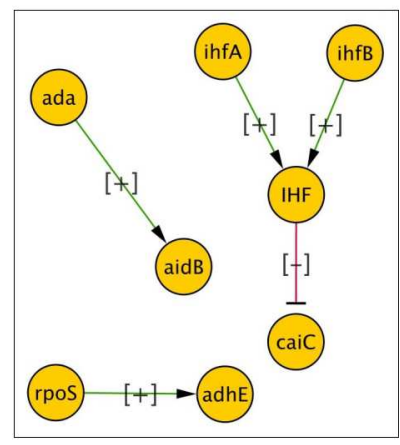

B

Figure 1: A regulatory network (A) mapped into an influence network $(\mathbf{B})$. Influences among molecules create an influence network; the arrows in the influence network represent a positive (+) or negative (-) influence.

The interactions of the influence network are described qualitatively as,,+- , and ?, where: + , represents a positive influence (e.g. activation of gene transcription, recognition of a gene promoter region, or formation of proteins complexes); -, a negative influence (e.g. inhibition of gene transcription, inactivation of a protein); and ?, a dual or complex regulation. Differential data issued from perturbation steady state experiments may be optionally provided and must represent qualitative $(+,-)$ concentration changes of some of the molecules in the network. It is important that during the perturbation experiment influence relations in the network remain constant. One type of concentration changes may be statistically significant mRNA-expression responses; described qualitatively as: + up-regulation, - down-regulation. We may also provide other reliable concentration changes issued from the literature or different experiments.

An influence network is analysed using the following consistency rule: "The variation of the concentration level of one molecule in the network must be explained by an influence received from at least one of its predecessors, different from itself, in the network". Checking the validity of this rule for an influence network alone, or for an influence network plus a set of reliable concentration changes, will be referred from now on as the consistency check process. The mathematical bases of the consistency rule are formally proven in [24], and [27]. In these studies the authors assumed that the concentration changes must correspond to changes between two stable conditions in the cell.

Let us intuitively explain the logic of the consistency rule in the simple influence network presented in Fig. 2. $A$ and $B$ may represent two proteins that activate the transcription of gene $C$. The consistency rule states that if $A$ and $B$ are both up-regulated (+) under certain condition, then $C$ must be up-regulated, i.e. a + prediction will be assigned to $C$ (Fig. 2A). Similarly, the concentration change of $C$ will be predicted as - if both, $A$ and $B$, were down-regulated.

When $A$ is up-regulated (+) and $B$ is down-regulated (-) the expression level of $C$ cannot be predicted, as both expression levels (up/down regulated) are possible for $C$ and do not contradict the consistency rule (Fig. 2B). Only if $C$ was a protein complex formed by proteins $A$ and $B$, we may predict the expression level of $C$ depending on the change in expression of the limiting former protein. To state this, we have extended the theory in $[24,27]$ in a context where the weakest takes it all concluding in a rule called the protein complex behaviour rule. This rule applies when the values of the concentrations of A and B are well separated (one of them is much smaller than the other). The details of this rule are provided in the Supplementary material.

A third situation may occur when all the molecules are observed, let us say, $A$ is up-regulated, $B$ is up-regulated, and $C$ is down-regulated. The consistency rule states that $C$ should be up-regulated; the experiment, however, shows the contrary. Thus, we arrive to a contradiction between the network and the experiment, also called inconsistent model (Fig. 2C). No predictions may be generated from 
an inconsistent model, yet, a region in the network is identified together with the expression data that created the conflict.

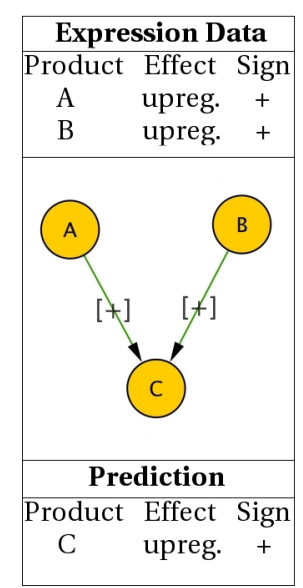

A

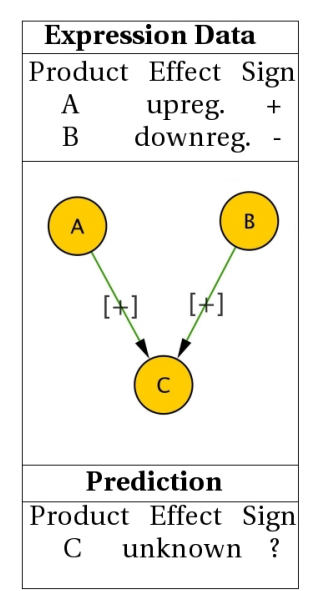

B

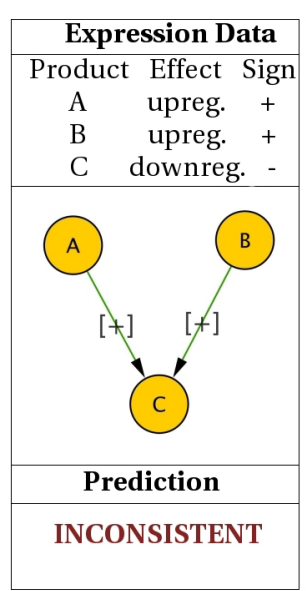

C

Figure 2: Examples explaining the consistency check process. A. Expression predictions when a set of consistent expression data is provided. B. Consistent expression data may not generate a new prediction. C. Expression data provided resulted inconsistent with the influence network.

An influence network consistent with expression data is a network where all the expression data is explained by the (consensual or not) fluctuation of some of the nodes in the network. In Fig. 2 we illustrated that depending on the expression data, we may obtain up to three different results: consistency and prediction, just consistency, or inconsistency. In order to evaluate the consistency of a large-scale network, we represent it as a system of qualitative constraints in $\{+,-, ?\}$, where each constraint relates a node with its direct predecessors. All constraints in the system should satisfy the consistency rule, and should not contradict any other. Computing the satisfiability of a large system of qualitative constraints is an NP-complete problem for even linear qualitative systems [10]. As classic methods of resolution do not allow to solve this kind of problems [28], we used an efficient representation based on binary decision diagrams for the set of solutions of a qualitative system proposed in [29]. The consistency of a large system of qualitative constraints is thus computed using Pyquali, a Python library devoted to solve huge systems of constraints in three values $(+,-, ?)$ that is based on the mentioned representation. As a result, it is possible to decide in less than one minute if a large-scale regulatory model is consistent with an initial dataset and to detect inconsistent regions in the model when needed.

In Fig. 3 we illustrate a flow-chart of the complete consistency check process. As input data we receive a qualitative influence network and a set of qualitative concentration changes. We build from this initial data a system of mathematical constraints, its consistency will be analysed afterwards using Pyquali. If the system is consistent, we predict new concentration changes that after being compared with other experiments may generate new inputs to the initial set of concentration sets, however, it is also possible that thanks to this comparison new nodes and edges in the influence network may be added. If the system is inconsistent, then the influence network must be corrected either by searching in the literature or by experimental results.

\subsection{Small example}

To understand how a consistent model may generate computational predictions, let us analyse in detail the consistency check process applied to a small region of the E. coli influence network. The influence network presented in Fig. 4 is analysed during the exponential-stationary growth shift. An initial dataset of concentration changes (obtained from the literature) was initially provided for fic, ihf $A$, and $\operatorname{ihf} B[16,3]$. From this initial data the analysis proceeds as follows. As fic is 


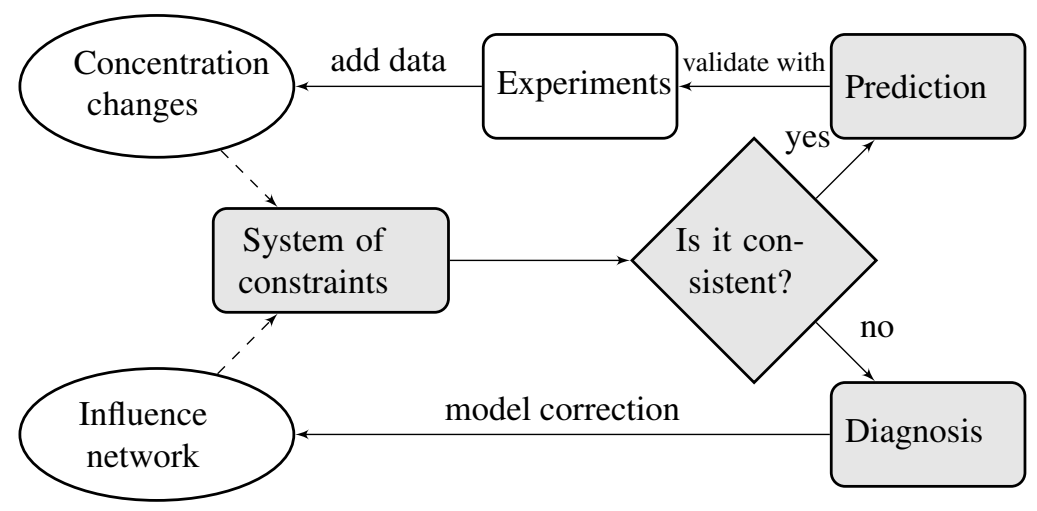

Figure 3: Consistency check process. (1) We build a system of constraints from an influence network with a set of concentration changes, (2) we check the consistency of the system, (3) if it is consistent and an initial dataset of concentration changes was provided, we may predict new concentration changes of the molecules in the network that after compared with real measurements may question the original dataset and model. If it is not consistent, we report the inconsistent region in order to correct the network or initial dataset. The shaded blocks represent the automatically calculated steps.

up-regulated, and it only receives an influence from the sigma factor RpoS, then RpoS is fixed to be up-regulated. The protein complex IHF is fixed as down-regulated after applying the protein complex behaviour rule deduced from observing the concentration of its former proteins and the metabolically stable behaviour of IHF in its dimeric form (see the Supplementary material). The gene transcribing one of its former proteins, ihf $B$, appears down-regulated; hence, it must receive a negative influence from one of its three predecessors in the network: IHF's influence is positive as it is down-regulated but it inhibits ihf B's expression; RpoS's influence is also positive as it was predicted to be up-regulated; only RpoD's value can be fixed to down-regulated (-), causing a negative influence over ihf $B$. The genes that receive a unique influence from RpoD, $\operatorname{crp}$ and $\operatorname{omp} A$, are fixed as down-regulated because of the negative influence coming from RpoD. No other alternatives nor contradictions appear in the fixed values of IHF, RpoS, RpoD, crp, and ompA; consequently, they are the computational predictions.

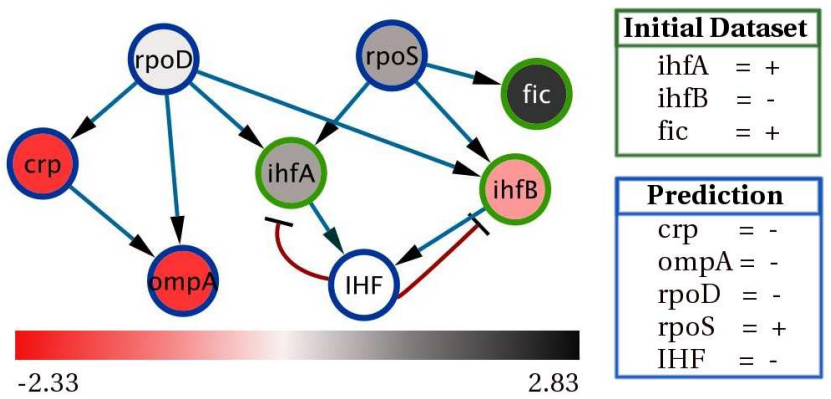

Figure 4: Consistency check process for 8 products of the E. coli regulatory network under the exponential-stationary growth shift. All transcriptional influences that each product receives appear in the network. The grey-red intensity of each product reflects the experimentally observed change in mRNA expression $\left(\log _{2}\right.$ ratio) during the studied condition. Products with a green border refer to those present in the initial dataset obtained from the literature, whereas products with a blue border refer to our computational predictions. Experimentally observed mRNA-expression changes agree with the computational predictions except for RpoD, where the predicted changes correspond to variations on the active protein and cannot be observed on mRNA expression levels.

Experimentally observed mRNA-expression changes [1] agree with the computational predictions except for RpoD, as shown in Fig. 4. The reason is that in our influence network, rpoD represents the active RpoD global sigma-factor that enhances the transcription of many E. coli genes by binding to RNA-polymerase. When we predict the change of rpoD as -, this means that the amount of active 
RpoD has been reduced which does not necessarily implies that its mRNA expression level is downregulated. During the entry to stationary phase, indeed, the Rsd (Regulator of Sigma D) protein forms a complex with RpoD preventing it to bind RNA-polymerase [20]; this is why rpoD decreases its level (its active concentration level) even if its mRNA expression level appears to be slightly up-regulated. This kind of contradictions between the computational predictions and mRNA expression data may help us to elucidate post-transcriptional mechanisms stemmed from Protein-Protein interactions that were initially absent in our model.

\section{Results}

\subsection{Construction of the network}

We constructed the influence network from the regulatory model of E. coli available in the RegulonDB database [26]. The influences in our network represented transcriptional, complex formation, and Sigma-gene regulations. The products of the network were genes, active proteins, and protein complexes. The information to reconstruct this model was extracted from the database on June 2007. In Fig. 5 we show the complete and reduced network.

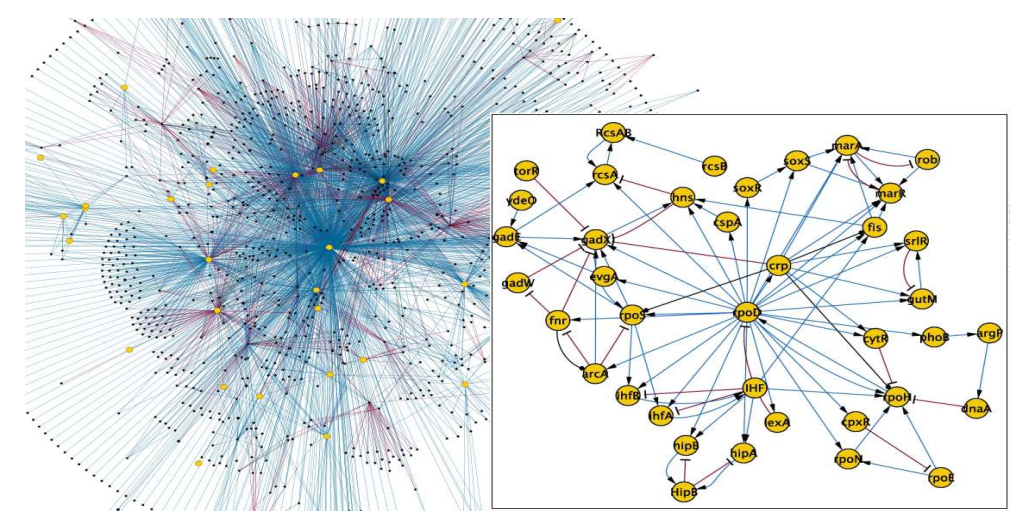

Figure 5: E. coli influence network (1763 products and 4491 interactions). A small region of it is presented to the right (39 products and 94 interactions). The products forming this region control most of the components of the bigger network.

\subsection{Consistency test and correction using one stress condition}

To start with the consistency check process, two initial data were provided: (i) E.coli influence network, and (ii) a set of 45 differentially expressed genes in the transition from exponential to stationary growth phase. This set of phenotypes was collected from the literature based on initial information provided in RegulonDB. The first result obtained was an inconsistency between the model and the experimental data. The inconsistent region, highlighted by the method, is the one shown in Fig. 6A. It represents the inhibition of transcription of the appY gene by the H-NS protein; no other transcrip-

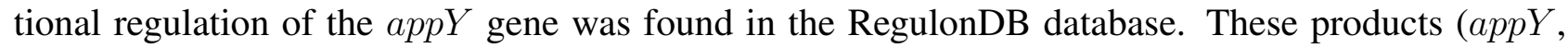
hns, and therefore H-NS) are however, shown to increase their levels in the exponential-stationary growth shift of the cell $[9,2]$. Hence, the source of the conflict may be in the model.

Searching in the primary literature, we found that the appY gene is induced during entry into stationary phase, and that during oxygen-limiting conditions the stationary-phase induction is partially dependent on ArcA [5]. The protein ArcA is activated via phosphorylation by the ArcB sensor under conditions of reduced respiration [19]; the signal which leads to the activation of ArcA during entry into stationary phase may be the deprivation of oxygen caused by an increase in cell density [5]. Based on these studies we corrected our influence network adding new interactions (see Fig. 6B), obtaining a model consistent with the experimental data. 


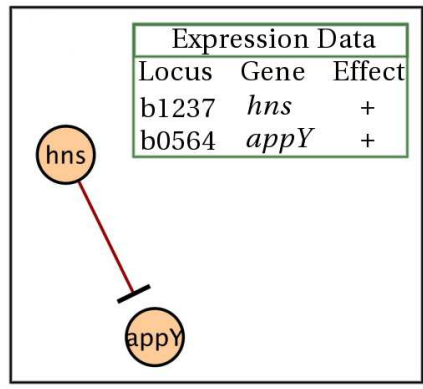

A

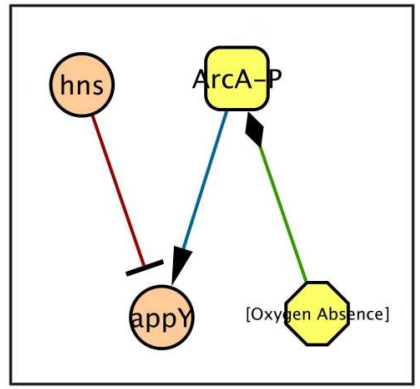

B

Figure 6: Diagnosis when an inconsistency between the model and data is found. A. The inhibition of gene app $Y$ by the hns product causes an inconsistency with the expression data related to the exponential-stationary growth shift. B. Correction of the inconsistency by adding a positive regulation from ArcA-P (phosphorylated protein ArcA) to appY; this regulation occurs in the absence of oxygen.

\subsection{Computational predictions and validation}

Once our network was consistent with the expression data provided for the exponential-stationary growth shift, we generated the computational predictions. From the 45 initial expression phenotypes, 526 changes in other components of the network during the same experimental condition were predicted. We characterized them into 12 functional groups using the DAVIS software provided in [8]. To validate our computational predictions, we obtained from the Many Microbe Microarray Database [12] a dataset of differentially expressed genes after 720 minutes of growth (stationary phase) in a rich medium [1]. This dataset was compared to our predictions (Fig. 7).

Expression profiling of this microarray dataset identified 926 genes that change significantly (2fold) in transcription in response to the growth shift from exponential to stationary phase. The 526 products, computationally predicted, could be classified into four categories: 131 agreed with significant expression changes; 32 had a predicted expression change in a direction opposite to that of the experimental data; 329 had a predicted expression change that was not found to be statistically significant in the experimental data; and for 34 products there was no expression data available (some products of the network were protein complexes and could not be compared to mRNA expression). Thus, of the $163(=131+32)$ significant differentially expressed genes that could be compared between the computational predictions and the experiment, 131 (or 80\% consensus) agreed. Only the $31 \%$ (coverage) of our predictions could be compared with 2-fold expression changes; therefore we performed the same analysis choosing different thresholds (1.5-fold, 0 fold). In this way, new consensus and coverage percentages were calculated showing that the higher the threshold is, the better is the consensus and - as expected - the worse is the coverage of the predicted data (Fig. 8).

The quality of our computational predictions is related to the experimental condition used to calculate them. Three important phases in the cell (exponential, early stationary-phase, late stationaryphase), appear in the exponential-stationary growth shift. During the shift among these phases, many molecules in the cell change their behaviour considerably. In our study we have chosen to compare the late stationary phase, when the number of cells does not change, with the late exponential growth phase, when the cell continues to grow exponentially. We believe that these two conditions represent instants in the cell where the genes and proteins do not significantly change their concentration. However, the 45 initial expression data may correspond to slightly different time points of the exponential phase and thus induce divergences in our computational predictions. In spite of this, a high percentage of our predictions was validated when compared with the microarray data.

\section{Discussion}

We have introduced an iterative method that checks the consistency between a regulatory model and expression data. To validate our approach we chose the E. coli transcriptional regulatory net- 

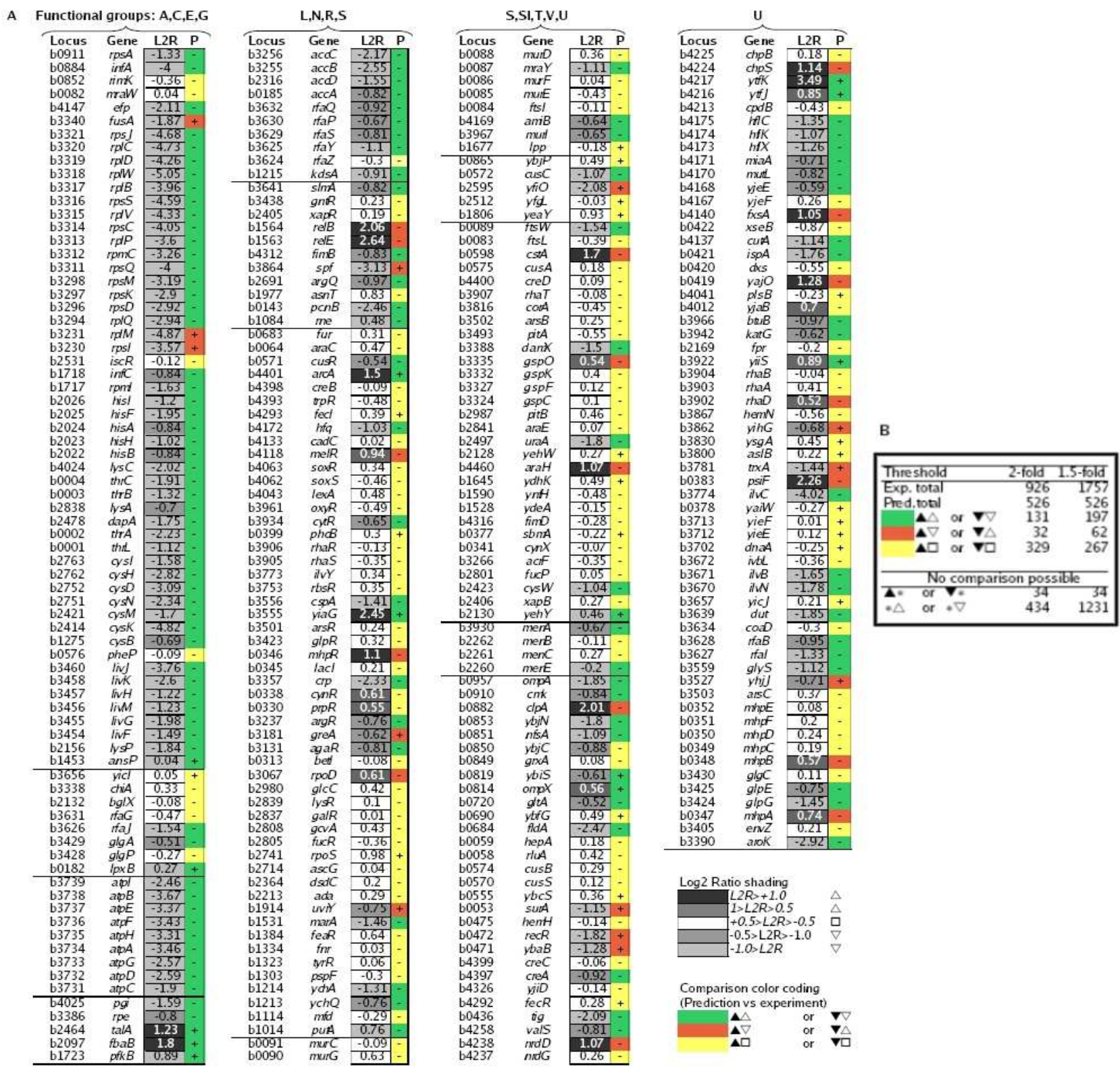

Figure 7: Table of observed vs. predicted gene-expression responses in the E.coli network under the exponential-stationary growth shift condition. A. The locus numbers, gene names, and the $\log _{2}$ ratio (L2R) of gene expression (exponential to stationary) are shown for some of the 526 predicted expression changes (+,-). Genes were divided by functional groups into: A, amino acids metabolism and biosynthesis; C, carbohydrates metabolism and biosynthesis; E, energy metabolism; G, glucose catabolism; L, lipid metabolism and biosynthesis; N, nucleic acids metabolism; R, regulatory function; S, cell structure; SI, signal peptides; T, transport; V, vitamin metabolism and biosynthesis; and U, unassigned. The L2R is shaded depending on the magnitude of the expression shift. Filled and open symbols indicate computational predictions and experimental data, respectively; squares indicate no change in gene expression; triangles indicate a change in expression, as well as the direction of change (up-regulated or down-regulated).B. Comparison between all predicted and observed expression changes. An $*$ symbol indicates either that our model did not predict a gene expression or that no expression data related to a gene in our model was found.

work obtained from the RegulonDB database. By using an expression dataset of 45 E. coli genes significantly expressed under the exponential-stationary growth shift, we corrected the model and predicted the outputs of microarray experiments with an $80 \%$ of agreement. This percentage is comparable to the one obtained by other methods working on a more complex regulatory model for $E$. coli $[7,6,11]$. After the comparison of our computational predictions with microarray measurements, some disagreements were detected. A reasonable explanation for these divergences resides on 


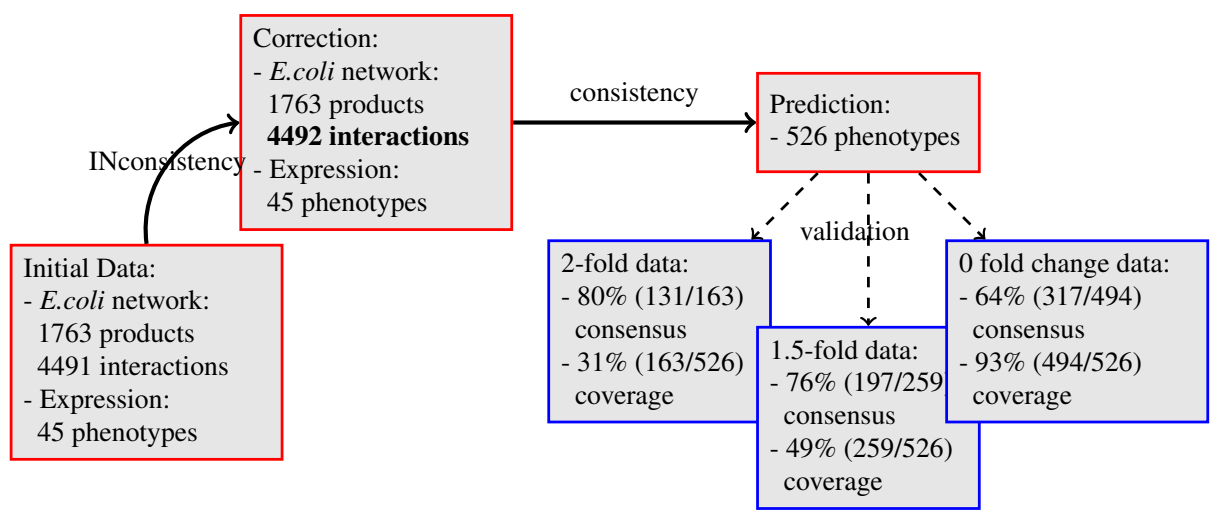

Figure 8: Results of the consistency check process applied to the E.coli transcriptional network using 45 phenotypes related to the exponential-stationary growth shift. We validated our computational predictions using the observations in a microarray dataset filtered with three thresholds. Consensus refers to the percentage of validated model predictions; coverage indicates the percentage of compared predictions.

our previously made assumption that some level of correlation exists between the transcription factor protein and the target gene expression without considering in detail the post-transcriptional effects; this problem was also reported in [15]. An example of this case of disagreement was illustrated in Fig. 4; nevertheless, we can still use this type of errors in our predictions to complete the regulatory model with post-transcriptional regulations. A second possible reason is the complexity of the exponentialstationary growth shift; this induces heterogeneity in the observations from different sources of the literature [14].

We illustrated that our automatized framework checks in short time the global consistency of a large-scale system of constraints, into which regulatory and expression knowledge can be represented. Computationally, the solution of this type of problems is not trivial, nevertheless we analysed the complete large-scale regulatory network of E.coli in less than one minute. Biologically speaking, we are able to integrate large-scale regulatory data and confront it to experimental observations in order to provide a diagnosis of regions in the network where the regulatory knowledge is contradictory with the expression data. Moreover, we compute a set of computational predictions that represent the invariant regions in the network that explain our expression data consistently. These results reflect important global configurations in a regulatory network that can be practically used to diagnose models and in a future to modulate their global behaviour. What we have shown for the E.coli network, can be applied to other networks and to different expression datasets.

\section{Supplementary material}

The supplementary material is available on-line at: http://www.irisa.fr/symbiose/projects/supplementary1.pdf.

\section{Acknowledgments}

We would like to thank Michel Le Borgne and Philippe Veber for their invaluable work implementing Pyquali. We also thank Daniel Kahn for very useful discussion. The work of the first autor was financially supported by CONICYT (Comisión Nacional de Investigación Científica y Tecnológica), Chile. This work was supported by the ANR program ANR-06-BYOS-0004.

\section{References}

[1] Allen, T., Herrgrd, M., Liu, M., Qiu, Y., Glasner, J., Blattner, F., and Palsson, B. (2003). Genome-scale analysis of the uses of the Escherichia coli genome: model-driven analysis of heterogeneous data sets. J Bacteriol, 185(21), 6392-9.

[2] Atlung, T., Sund, S., Olesen, K., and Brndsted, L. (1996). The histone-like protein H-NS acts as a transcriptional repressor for expression of the anaerobic and growth phase activator AppY of Escherichia coli. J Bacteriol, 178(12), 3418-3425. 
[3] Aviv, M., Giladi, H., Schreiber, G., Oppenheim, A., and Glaser, G. (1994). Expression of the genes coding for the Escherichia coli integration host factor are controlled by growth phase, rpoS, ppGpp and by autoregulation. Mol Microbiol, 14(5), 1021-31.

[4] Bansal, M., Belcastro, V., Ambesi-Impiombato, A., and di Bernardo, D. (2007). How to infer gene networks from expression profiles. Mol Syst Biol., 3(78).

[5] Brondsted, L. and Atlung, T. (1996). Effect of growth conditions on expression of the acid phosphatase (cyx-appA) operon and the appY gene, which encodes a transcriptional activator of Escherichia coli. J Bacteriol, 178(6).

[6] Covert, M. and Palsson, B. (2002). Transcriptional regulation in constraints-based metabolic models of Escherichia coli. J biol chem, 277(31), $28058-28064$.

[7] Covert, M., Knight, E., Reed, J., et al. (2004). Integrating high-throughput and computational data elucidates bacterial networks. Nature, 429(6987), 92-6.

[8] Dennis, G., Sherman, B., Hosack, D., et al. (2003). VID: Database for Annotation, Visualization, and Integrated Discovery. Genome Biol, 4.

[9] Dersch, P., Schmidt, K., and Bremer, E. (1993). Synthesis of the Escherichia coli K-12 nucleoid-associated DNA-binding protein H-NS is subjected to growth-phase control and autoregulation. Mol Microbiol., 8(5), 875-89.

[10] Dormoy, J. (1988). Controlling qualitative resolution. In 7th National Conference on Artificial Intelligence, AAAI'88, Saint Paul, Min., USA.

[11] Edwards, J. and Palsson, B. (2000). The Escherichia coli MG1655 in silico metabolic genotype: its definition, characteristics, and capabilities. PNAS, 97(10), 5528-33.

[12] Faith, J., Driscoll, M., Fusaro, V., Cosgrove, E., Hayete, B., Juhn, F., Schneider, S., and Gardner, T. (2007a). Many microbe microarrays database: uniformly normalized affymetrix compendia with structured experimental metadata. Nucleic Acids Res.

[13] Faith, J. J., Hayete, B., Thaden, J. T., et al. (2007b). Large-scale mapping and validation of Escherichia coli transcriptional regulation from a compendium of expression profiles. PLoS Biol, 5(1), e8.

[14] Gutierrez-Rios, R. M., Rosenblueth, D. A., Loza, J. A., et al. (2003). Regulatory network of Escherichia coli: consistency between literature knowledge and microarray profiles. Genome Res, 13(11), 2435-2443.

[15] Herrgard, M., Covert, M., and Palsson, B. (2003). Reconciling gene expression data with known genome-scale regulatory network structures. Genome Res, 13(11).

[16] Hiratsu, K., Shinagawa, H., and Makino, K. (1995). Mode of promoter recognition by the Escherichia coli RNA polymerase holoenzyme containing the sigma S subunit: identification of the recognition sequence of the fic promoter. Mol Microbiol., 18(5).

[17] Ideker, T., Thorsson, V., Ranish, J., et al. (2001a). Integrated genomic and proteomic analyses of a systematically perturbed metabolic network. Science, 292(5518).

[18] Ideker, T., Galitski, T., and Hood, L. (2001b). A new approach to decoding life: systems biology. Annu Rev Genomics Hum Genet., 2, 343-72.

[19] Iuchi, S., Chepuri, V., Fu, H. A., et al. (1990). Requirement for terminal cytochromes in generation of the aerobic signal for the arc regulatory system in Escherichia coli: study utilizing deletions and lac fusions of cyo and cyd. J Bacteriol, 172(10).

[20] Jishage, M. and Ishihama, A. (1998). A stationary phase protein in Escherichia coli with binding activity to the major sigma subunit of RNA polymerase. PNAS, 95(9).

[21] Joyce, A. R. and Palsson, B. O. (2006). The model organism as a system: integrating 'omics' data sets. Nat Rev Mol Cell Biol, 7(3), 198-210.

[22] Kitano, H. (2002). Systems biology: A brief overview. Science, 295(5560), 1662-1664.

[23] Palsson, B. (2000). The challenges of in silico biology. Nature Biotechnology, 18.

[24] Radulescu, O., Lagarrigue, S., Siegel, A., et al. (2006). Topology and static response of interaction networks in molecular biology. $J$ R Soc Interface, 3(6), 185-96.

[25] Rawool, S. and Venkatesh, K. (2007). Steady state approach to model gene regulatory networks-simulation of microarray experiments. Biosystems, 90(3), 636-55.

[26] Salgado, H., Gama-Castro, S., Peralta-Gil, M., and al. (2006). RegulonDB (version 5.0): Escherichia coli K-12 transcriptional regulatory network, operon organization, and growth conditions. Nucleic Acids Res, 34(Database issue), D394-7.

[27] Siegel, A., Radulescu, O., Borgne, M. L., Veber, P., Ouy, J., and Lagarrigue, S. (2006). Qualitative analysis of the relation between DNA microarray data and behavioral models of regulation networks. Biosystems, 84(2), 153-74.

[28] Travé-Massuyès, L. and Dague, P. (2003). Modèles et raisonnements qualitatifs. Hermes Sciences, Paris.

[29] Veber, P., Borgne, M. L., Siegel, A., Lagarrique, S., and Radulescu, O. (2004/2005). Complex qualitative models in biology: A new approach. Complexus, 2(3-4). 